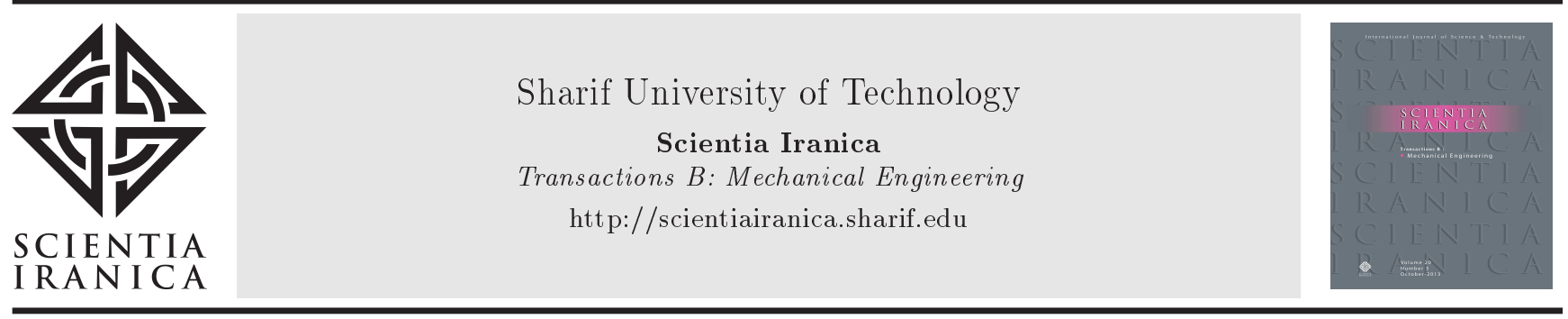

\title{
Evolution of thin liquid film for Newtonian and power-law non-Newtonian fluids
}

\author{
R. Nasehi ${ }^{a, *}$ and E. Shirani ${ }^{b}$ \\ a. Department of Mechanical Engineering, Isfahan University of Technology, Isfahan, P.O. Box 84156-83111, Iran. \\ b. Foolad Institute of Technology, Fooladshahr, Isfahan, P.O. Box 84916-63763, Iran. \\ Received 17 May 2016; accepted 7 January 2017
}

\section{KEYWORDS}

Analytical relation;

Newtonian fluid;

Non-Newtonian fluid;

Power-law model;

Rayleigh-Taylor

instability;

Thin liquid film.

\begin{abstract}
Analytical relations for slow-motion thin liquid films bounded by a fixed wall and free surface for Newtonian and non-Newtonian fluids are obtained in this work. Assuming long-wave approximation, the momentum and continuity equations for thin liquid films of power-law fluids are simplified and solved analytically to derive the evolution equation of thin liquid films. The evolution equation is derived for two- and threedimensional cases. A relation for evolution of thin films is obtained for a simple case in which the liquid film is supported from below by a solid surface and subjected to gravity and constant surface tension forces. This evolution equation of thin film has been solved numerically in order to compare the behavior of Newtonian and non-Newtonian liquids for different Bond numbers. It is shown that the power-law model at low and high strain rates is invalid and it affects the results. The Rayleigh-Taylor instability is another subject that is studied in this work. This interesting phenomenon is investigated by numerically solving the evolution equation for different Bond numbers. The results show that the evolution of the free surface thin film for pseudo plastic fluids is different from that for Newtonian and dilatant fluids.

(C) 2018 Sharif University of Technology. All rights reserved.
\end{abstract}

\section{Introduction}

Competing with numerical methods, analytical solution of governing equations of fluid flows has attracted the attention of many scientists and engineers. Deriving analytical solutions for fluid flows is challenging in most cases. Thus, application of numerical methods is inevitable; however, numerical methods have their own limitations, such as inaccuracy, numerical instability, and computational costs. Yet, solving a single partial differential equation, which well expresses the fluid

\footnotetext{
*. Corresponding author. Tel.: +983133915266; Fax: +983133912628

E-mail addresses: ramin.nasehi@gmail.com (R. Nasehi); eshirani@ictp.it (E. Shirani)
}

doi: $10.24200 /$ sci.2017.4320 flow, is far preferred over solving a system of equations, including continuity and momentum equations, due to limitations of numerical methods. In cases with thin film flows, it is possible to track the interface by a single partial differential equation, which is obtained from governing equations using some simplifying assumptions. The aim of this work is to find a single general equation for tracking the non-Newtonian thin film.

Liquid film is a layer of fluid that is supported by a solid plate and bounded by an interface between the plate and another fluid. Laminar flow of thin liquid films is one of the interesting subjects in fluid mechanics as it is applicable in biophysics, physics, and engineering, in addition to being an interesting natural phenomenon. From industrial point of view, this subject is applicable in slivering, optical coating, lubricating, adhesives, common liquids such as water 
and oil, more complex materials such as polymer solutions and melts, and mixtures of different fluids. Films subjected to various mechanical and thermal influences show interesting phenomena such as wave propagation, wave steepening, fingering, and spreading. Therefore, scientists and engineers pay significant attention to this topic.

Liquid film in which the perturbed wavelength at the interface is much larger than its mean thickness is considered as thin liquid film. Under such conditions, by using long-wave approximation and simplifying continuity and momentum equations for slow flow of Newtonian incompressible viscous fluid, one achieves a single equation governing film evolution. For the simplest case in which the film is supported from below by a solid surface and is subjected to constant surface tension forces and gravity is the only body force, the evolution of thin films is expressed by a single fourth order equation [1]. When the liquid film is positioned under a plate, except for small Bond numbers (relatively large surface tension force to gravitational one), the film is unstable and Rayleigh-Taylor instability occurs [2]. The Rayleigh-Taylor instability has been studied by Yiantsios and Higgins [3,4] for cases that a layer of fluid with lower density is positioned under a semi-infinite fluid. They stated that "instability leads to a spectrum of steady-state interfacial shapes characterized by film rupture and formation of pendant drops, such that the gravity and interfacial forces are in balance." Oron and Rosenau [5] also studied this phenomenon for thin liquid film under a rigid plane. Fermigier et al. [6] investigated the problem both experimentally and theoretically. In their work, the formation of patterns of different symmetries and transition between patterns and droplet detachment were observed. Ruyer-Quil and Mannevilla [7] represented a high order model for flows on inclined planes that was capable to handle strong nonlinear regimes. More information about thin liquid films for Newtonian fluids is provided in [2].

Study of low-velocity non-Newtonian fluid flows is important since some of the fluids used in industry as well as in laboratories are non-Newtonian. Houseman and Molnar [8] studied Rayleigh-Taylor instability to find out how stress-dependent viscosity affected convective instability associated with thickening of cold, dense lithosphere beneath mountain belts. They showed that "the non-linearity in constitutive law effects a delay in the convective thinning that is not seen when Newtonian viscosity is assumed." Fomin et al. [9] investigated rimming flows of power-law fluid in the inner side of horizontally rotating cylinder. Using lubrication theory, they showed that when the mass of non-Newtonian fluid exceeded a certain value or the rotation speed was less than a certain limit, a hydraulic jump would occur. They determined the height and lo- cation of the hydraulic jump. Perazzo and Gratton [10] studied the fingering instability of a slow flow of nonNewtonian liquid on an inclined surface. In another work [11], they studied a family of traveling waves of a flow of power-law fluid on an inclined plate using long wave approximation and showed that there were exactly seventeen different kinds of solutions. Balmforth et al. [12] investigated surface-tension-driven fingering of a thin layer of viscoplastic fluid. They obtained traveling wave solutions and applied a linear stability analysis to model finger growth. They demonstrated qualitative agreement between their numerical results and experiment. Miladinova et al. [13] studied slow flow of power-law non-Newtonian fluid on an inclined plate in a periodic domain. They illustrated that the free surface evolution of non-Newtonian fluids was similar to that of Newtonian fluids while the shape and amplitude of the permanent wave were influenced by non-Newtonian behavior. Myers [14] compared the results of three models of non-Newtonian fluid (Ellis, power-law, and Carreau) for free surface flow and flow in a channel. Perazzo and Gratton [15] presented exact solutions for two-dimensional steady flows on an inclined plate using power-law model. Their analysis did not include surface tension effect. Heining and Aksel [16] studied a power-law fluid flowing down an inclined wall with sinusoidal topography. They showed that increasing the steepness of the wall would increase the importance of non-linear effects. They also illustrated that shear thickening and shear thinning flows had different behaviors. $\mathrm{Hu}$ and Kieweg [17] studied the effect of surface tension force on thin film flows of a power-law liquid on an inclined plate. They found that the surface tension force affected both the spreading speed and the shape of two-dimensional spreading profile.

In this work, the analytical relation for thin non-Newtonian liquid films is investigated. At first, assuming long-wave approximation, the momentum and continuity equations for thin liquid films of powerlaw fluids are simplified. Then, considering appropriate boundary conditions, the system of equations is analytically solved in order to derive the evolution equation of thin liquid films for power-law non-Newtonian fluids. This evolution equation is highly non-linear and is derived for two- and three-dimensional cases. Considering the simplest case in which the film is under a solid surface and subjected to the influence of gravity and constant surface tension forces, the evolution of thin films is investigated. The stability properties of a uniform film perturbed by small periodic disturbances are studied and the evolution equation of the thin film is numerically solved in order to compare the behavior of Newtonian and non-Newtonian liquid films for different Bond numbers. The RayleighTaylor instability is another subject that is studied 
in this work. Here, the gravity force acts as a source of instability in the liquid films' motion. This phenomenon is investigated by numerically solving the derived evolution equation for different Bond numbers and the pseudo plastic, dilatant, and Newtonian fluid flows have been investigated and discussed.

\section{Evolution equation}

Long-wave approximation is considered and applied to slow motion of non-Newtonian incompressible viscous liquid film on a plate. The continuity and momentum equations are:

$$
\begin{aligned}
& \nabla \cdot V=0, \\
& \rho \frac{D V}{D t}=-\nabla p+\eta(s) \nabla^{2} V+2 D . \nabla \eta-\nabla \varphi,
\end{aligned}
$$

where, $V=(u, v, w)$ is the velocity field in the liquid film; $t$ is time; $p$ is pressure; $\nabla \equiv(\partial / \partial x, \partial / \partial y, \partial / \partial z)$; $\nabla^{2} \equiv\left(\partial^{2} / \partial x^{2}, \partial^{2} / \partial y^{2}, \partial^{2} / \partial z^{2}\right) ; D / D t$ is the substantial derivatives; $\varphi$ is potential function, which determines the conservative forces, such as gravity, centrifugal, or van der Waals; and $\eta(s)$ is the fluid viscosity, which is a function of the strain rate, $s$. The function $\eta(s)$ varies depending on the non-Newtonian models. In this work, only the power-law model is considered. Therefore, $\eta(s)$ is:

$$
\eta(s)=K s^{(n-1) / 2},
$$

where, $K$ is a constant, $n$ is the power-law index, and $s$ is obtained as:

$$
s=-4 I I_{D}=2 \operatorname{tr}\left(D^{2}\right),
$$

where, $D=1 / 2\left(\nabla V+\nabla V^{T}\right)$ is the strain rate tensor.

Assuming no-penetration and no-slip boundary conditions on the solid surface, we have:

$$
w=0, \quad u=0, \quad v=0 .
$$

At the fluid free surface $z=h(x, y, t)$, the boundary conditions in the vector form are:

$$
h_{t}+V . \nabla h \sim w=0,
$$

and:

$$
\tau . n=-2 \widetilde{H} \sigma n+\nabla_{s} \sigma+\pi,
$$

where, $\tau$ is the stress tensor of the liquid; $n$ is the normal unit vector of the film surface; $\nabla_{s}$ is the surface gradient with respect to the interface; $\pi$ is the external force on the film surface with the components $\left(\pi_{3}, \pi_{1}, \pi_{2}\right)$ in the normal and tangential directions to the film surface; and $\widetilde{H}$ is the mean curvature of the interface determined by:

$$
\begin{aligned}
2 \widetilde{H} & =\nabla \cdot n \\
& =-\frac{h_{x x}\left(1+h_{y}^{2}\right)+h_{y y}\left(1+h_{x}^{2}\right)-2 h_{x} h_{y} h_{x y}}{\left(1+h_{x}^{2}+h_{y}^{2}\right)^{3 / 2}},
\end{aligned}
$$

where, subscripts denote partial differentiation with respect to $x$ and $y$. Eq. (6) is the kinematic boundary condition in the absence of interface mass transfer and represents the balance between the normal component of the liquid velocity at the interface and the velocity of the interface itself. Eq. (7) constitutes the balance of the stresses in the absence of interfacial mass transfer at the interface.

By projecting Eq. (7) on the normal and tangential directions with respect to the film surface, we have Eq. (9) as shown in Box I.

Assuming a film with mean thickness, $d$, we introduce an appropriate scale for thin films. The length scales in lateral directions $x$ and $y$ are defined by wavelength $\lambda$ of disturbance on the film surface, as it is seen in Figure 1. Therefore, thin films refer to the films where $\lambda$ is much larger than $d$, that is:

$$
\varepsilon=\frac{d}{\lambda} \ll 1
$$

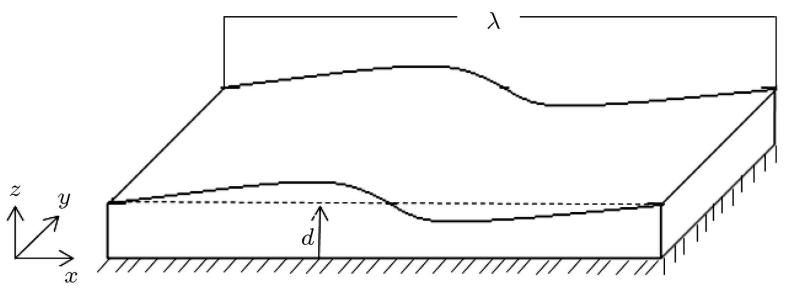

Figure 1. Flow configuration and coordinate system.

$$
\begin{aligned}
& \frac{2 \eta\left(\left.s\right|_{z=h}\right)\left[u_{x}\left(h_{x}^{2}-1\right)+v_{y}\left(h_{y}^{2}-1\right)+h_{x} h_{y}\left(u_{y}+v_{x}\right)-h_{x}\left(u_{z}+w_{x}\right)-h_{y}\left(v_{z}+w_{y}\right)\right]}{\left(1+h_{x}^{2}+h_{y}^{2}\right)}=p+\left(2 \widetilde{H} \sigma+\pi_{3}\right), \\
& \eta\left(\left.s\right|_{z=h}\right)\left[\left(u_{z}+w_{x}\right)\left(1-h_{x}^{2}\right)-h_{x} h_{y}\left(v_{z}+w_{y}\right)-h_{y}\left(u_{y}+v_{x}\right)-2 h_{x}\left(u_{x}-w_{z}\right)\right]=\left(\pi_{1}+\sigma_{x}\right)\left(1+h_{x}^{2}+h_{y}^{2}\right)^{1 / 2}, \quad(9 \\
& \eta\left(\left.s\right|_{z=h}\right)\left[-\left(u_{z}+w_{x}\right) h_{x} h_{y}+\left(v_{z}+w_{y}\right)\left(1-h_{y}^{2}\right)-h_{x}\left(u_{y}+v_{x}\right)-2 h_{y}\left(v_{y}-w_{z}\right)\right]=\left(\pi_{2}+\sigma_{y}\right)\left(1+h_{x}^{2}+h_{y}^{2}\right)^{1 / 2} .
\end{aligned}
$$


By normalizing the $z$-coordinate (normal to the solid surface) with $d$ and $x$-, $y$-coordinates with $\lambda$, no rapid variations occur as $\varepsilon \rightarrow 0$. The dimensionless velocities in the $x_{-}, y^{-}$, and $z$-directions are defined as:

$$
U=\frac{u}{U_{0}}, \quad V=\frac{v}{U_{0}}, \quad W=\frac{w}{\varepsilon U_{0}},
$$

where, $V=(U, V, W)$ is the dimensionless velocity field in the liquid film and $U_{0}$ is the characteristic velocity. One of the choices is $U_{0}=\eta_{0} / \rho d$, which is known as 'viscous velocity', where $\eta_{0}$ is the characteristic viscosity. Based on power-law model, we have:

$$
\eta_{0}=N . K\left(\frac{U_{0}}{d}\right)^{n-1}
$$

where, $N$ is a constant.

If $\lambda / U_{0}$ is considered as a time scale, the normalized time will be:

$$
T=\frac{\varepsilon U_{0} t}{d}
$$

The dimensionless interfacial stresses, body-force potential, pressure, and strain rate are defined as:

$$
\begin{aligned}
& \left(\Pi_{1}, \Pi_{2}, \varepsilon \Pi_{3}\right)=\frac{d}{\eta_{0} U_{0}}\left(\pi_{1}, \pi_{2}, \pi_{3}\right), \\
& (\Phi, P)=\frac{\varepsilon d}{\eta_{0} U_{0}}(\varphi, p), \quad S=\left(\frac{d}{U_{0}}\right)^{2} s .
\end{aligned}
$$

$\Lambda$ denotes the dimensionless viscosity and is defined as:

$$
\Lambda=\frac{\eta(s)}{\eta_{0}} .
$$

Substituting the dimensionless variables into the governing equations (1) and (2) and Eqs. (5)-(8), we have:

$$
\begin{aligned}
\varepsilon \operatorname{Re}( & \left.U_{T}+U U_{X}+V U_{Y}+W U_{Z}\right)=-P_{X} \\
& +\Lambda\left[\varepsilon^{2}\left(U_{X X}+U_{Y Y}\right)+U_{Z Z}\right] \\
& +\left(\frac{\partial \Lambda}{\partial S}\right)\left[2 \varepsilon^{2} U_{X} S_{X}+\varepsilon^{2}\left(U_{Y}+V_{X}\right) S_{Y}\right. \\
& \left.+\left(U_{Z}+\varepsilon^{2} W_{X}\right) S_{Z}\right]-\Phi_{X}, \\
\varepsilon \operatorname{Re}( & \left.V_{T}+U V_{X}+V V_{Y}+W V_{Z}\right)=-P_{Y} \\
& +\Lambda\left[\varepsilon^{2}\left(V_{X X}+V_{Y Y}\right)+V_{Z Z}\right] \\
& +\left(\frac{\partial \Lambda}{\partial S}\right)\left[\varepsilon^{2}\left(U_{Y}+V_{X}\right) S_{X}+2 \varepsilon^{2} V_{Y} S_{Y}\right. \\
& \left.+\left(V_{Z}+\varepsilon^{2} W_{Y}\right) S_{Z}\right]-\Phi_{Y}
\end{aligned}
$$

$$
\begin{aligned}
\varepsilon^{3} \operatorname{Re}( & \left.W_{T}+U W_{X}+V W_{Y}+W W_{Z}\right)=-P_{Z} \\
& +\varepsilon^{2} \Lambda\left[\varepsilon^{2}\left(W_{X X}+W_{Y Y}\right)+W_{Z Z}\right] \\
& +\varepsilon^{2}\left(\frac{\partial \Lambda}{\partial S}\right)\left[\left(U_{Z}+\varepsilon^{2} W_{X}\right) S_{X}\right. \\
& \left.+\left(V_{Z}+\varepsilon^{2} W_{Y}\right) S_{Y}+2 W_{Z} S_{Z}\right]-\Phi_{Z}
\end{aligned}
$$

The boundary conditions are as shown in Box II, where, $H=h / d$ is normalized film thickness, $\Sigma=$ $\varepsilon \sigma / \eta_{0} U_{0}$ is dimensionless surface tension, $\operatorname{Re}=$ $\rho U_{0} d / \eta_{0}$ is the Reynolds number, and $C a^{-1}=\sigma / \eta_{0} U_{0}$ is inverse of the capillary number.

It should be mentioned that the inertia terms in the momentum equations, measured by $\varepsilon R e$, are one order of magnitude smaller than the viscous terms. Also, the effect of surface tension, measured by $\varepsilon^{3} \mathrm{Ca}^{-1}$, is two orders of magnitude smaller than the inertia terms and can be neglected. Since the effects of surface tension on the revolution of thin film are retained, it is essential to assume that the capillary effects are strong compared with the viscosity effects and:

$$
C a^{-1}=\beta \varepsilon^{-3} \text {. }
$$

Under this assumption, Re and $C a^{-1}=O(1)$ as $\varepsilon \rightarrow 0$.

In order to solve the system of equations, the perturbation theory is used. Therefore, the perturbation series of dependent variables in terms of $\varepsilon$ are as follows:

$$
\begin{aligned}
& U=U^{(0)}+\varepsilon U^{(1)}+\varepsilon^{2} U^{(2)}+\cdots, \\
& V=V^{(0)}+\varepsilon V^{(1)}+\varepsilon^{2} V^{(2)}+\cdots, \\
& W=W^{(0)}+\varepsilon W^{(1)}+\varepsilon^{2} W^{(2)}+\cdots, \\
& P=P^{(0)}+\varepsilon P^{(1)}+\varepsilon^{2} P^{2}+\cdots .
\end{aligned}
$$

Substituting Eqs. (18) and (19) into system of equations and sorting them with respect to parameter $\varepsilon$, we obtain the governing system in terms of $\varepsilon$. We consider initial solution of thin film evolution; therefore, the governing equations become:

$$
\begin{aligned}
& U_{X}+V_{Y}+W_{Z}=0 \\
& -P_{X}+\Lambda U_{Z Z}+\left(\frac{\partial \Lambda}{\partial S}\right) U_{Z} S_{Z}-\Phi_{X}=0 \\
& -P_{Y}+\Lambda V_{Z Z}+\left(\frac{\partial \Lambda}{\partial S}\right) V_{Z} S_{Z}-\Phi_{Y}=0 \\
& P_{Z}+\Phi_{Z}=0
\end{aligned}
$$

The boundary conditions at $Z=0$ are:

$$
W=0, \quad U=0, \quad V=0,
$$




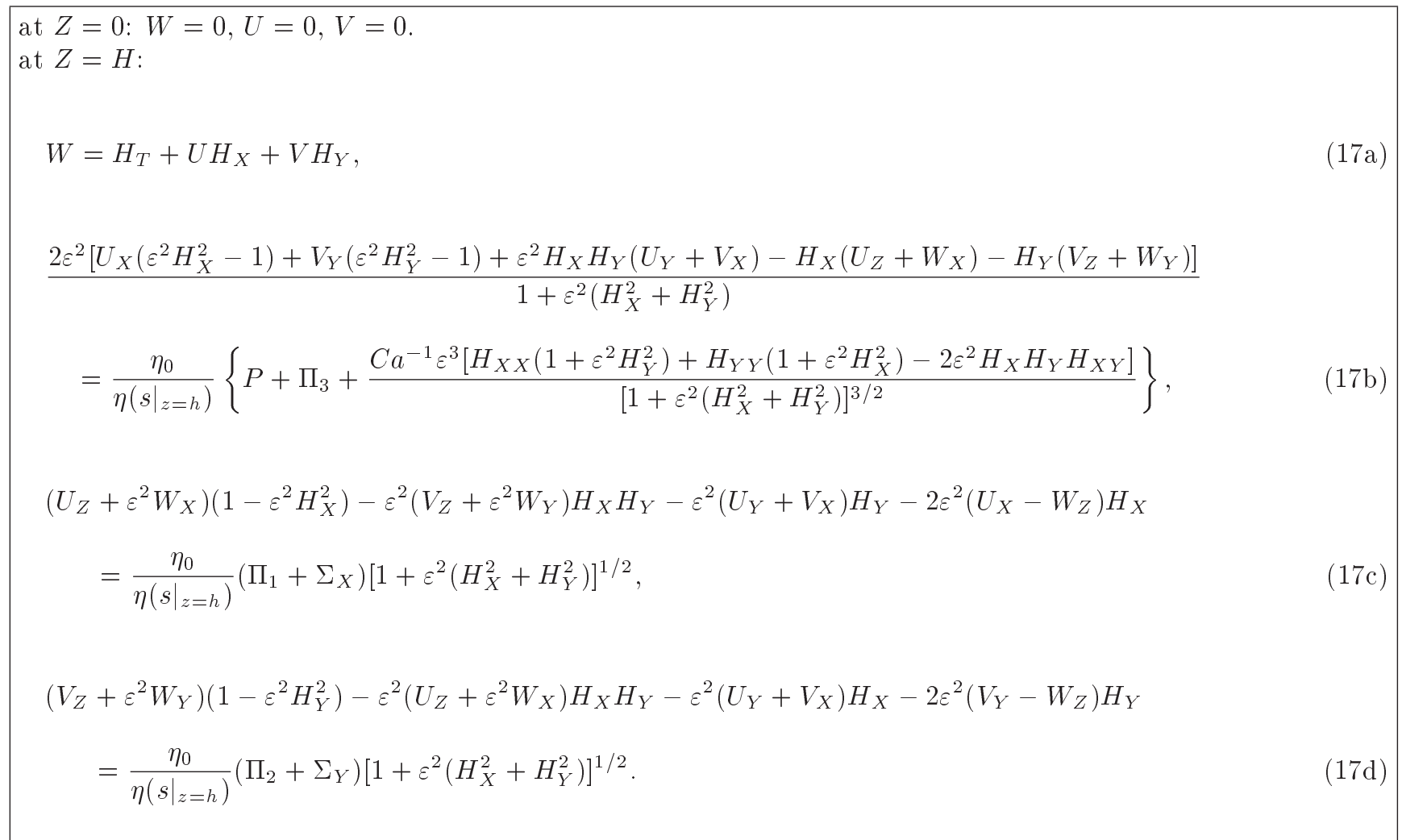

and at $Z=H$ are:

$$
\begin{aligned}
& H_{T}+U H_{X}+V H_{Y}-W=0, \\
& P=-\Pi_{3}-\beta\left(H_{X X}+H_{Y Y}\right), \\
& U_{Z}=\frac{1}{\Lambda\left(\left.S\right|_{Z=H}\right)}\left(\Pi_{1}+\Sigma_{X}\right), \\
& V_{Z}=\frac{1}{\Lambda\left(\left.S\right|_{Z=H}\right)}\left(\Pi_{2}+\Sigma_{Y}\right) .
\end{aligned}
$$

The continuity equation, Eq. (20), is integrated along $Z$-direction from 0 to $H(X, Y, T)$ and Eqs. (22) and (23a) are used along with it; the result of the integration is in a convenient form of kinematic condition and warrants the conservation of mass in the domain:

$$
H_{T}+\frac{\partial}{\partial X} \int_{0}^{H} U d Z+\frac{\partial}{\partial Y} \int_{0}^{H} V d Z=0 .
$$

In order to achieve the evolution equation for a powerlaw liquid film, the governing equations are solved to obtain $U$ and $V$. Then, integrating over the film thickness and substituting them into Eq. (24), we will achieve our goal to obtain the evolution equation of the thin film.

In order to find $U$ and $V$, Eqs. (21a) to (21c) are solved with appropriate boundary conditions expressed in Eqs. (23). $\Lambda$ is determined based on the power-law model, which is a function of the strain rate. Using the dimensionless parameters, the perturbation series, and Eqs. (3), (4), and (15), we have:

$$
\Lambda=\left[U_{Z}^{2}+V_{Z}^{2}\right]^{(n-1) / 2} \text {. }
$$

By substituting Eq. (25) into the system of equations and after performing some mathematical steps, which are described in the Appendix, we have:

$$
\begin{aligned}
U(Z)= & \frac{A^{X}}{\left(A^{X}\right)^{2}+\left(A^{Y}\right)^{2}} \frac{n}{n+1} \\
& \left\{\left[\left(A^{X} Z+B\right)^{2}+\left(A^{Y} Z+B^{\prime}\right)^{2}\right]^{(n+1) / 2 n}\right. \\
- & {\left.\left[(B)^{2}+\left(B^{\prime}\right)^{2}\right]^{(n+1) / 2 n}\right\} } \\
& -\frac{A^{Y}\left(\left|A^{X} B^{\prime}-A^{Y} B\right|\right)^{(n+1) / n}}{\left[\left(A^{X}\right)^{2}+\left(A^{Y}\right)^{2}\right]^{(3 n+1) / 2 n}} \\
& \left\{R_{(Z)}{ }_{2} F_{1}\left(\frac{1}{2}, \frac{-1+n}{2 n} ; \frac{3}{2} ;-R(Z)^{2}\right)\right. \\
& \left.-R_{0}{ }_{2} F_{1}\left(\frac{1}{2}, \frac{-1+n}{2 n} ; \frac{3}{2} ;-R_{0}^{2}\right)\right\},
\end{aligned}
$$




$$
\begin{aligned}
& V(Z)= \frac{A^{Y}}{\left(A^{X}\right)^{2}+\left(A^{Y}\right)^{2}} \frac{n}{n+1} \\
&\left\{\left[\left(A^{X} Z+B\right)^{2}+\left(A^{Y} Z+B^{\prime}\right)^{2}\right]^{(n+1) / 2 n}\right. \\
&\left.-\left[(B)^{2}+\left(B^{\prime}\right)^{2}\right]^{(n+1) / 2 n}\right\} \\
&+\frac{A^{X}\left(\left|A^{X} B^{\prime}-A^{Y} B\right|\right)^{(n+1) / n}}{\left[\left(A^{X}\right)^{2}+\left(A^{Y}\right)^{2}\right]^{(3 n+1) / 2 n}} \\
&\left.-R_{0}{ }_{2} F_{1}\left(\frac{1}{2}, \frac{-1+n}{2 n} ; \frac{3}{2} ;-R_{0}^{2}\right)\right\} \\
& W=- \int_{0}^{Z}\left(U_{X}+F_{1}\left(\frac{1}{2}, \frac{-1+n}{2 n} ; \frac{3}{2} ;-R(Z)^{2}\right)\right. \\
& P=-\Pi_{3}-\beta\left(H_{X X}+H_{Y Y}\right),
\end{aligned}
$$

where $A^{X}, B^{X}, A^{Y}, B^{Y}, B, B^{\prime}, R(Z)$, and $R_{0}$ are used to abbreviate the equations and are defined as:

$$
\begin{aligned}
& A^{X}=(P+\Phi)_{X}, \quad B^{X}=\left(\Pi_{1}+\Sigma_{X}\right), \\
& A^{Y}=(P+\Phi)_{Y}, \quad B^{Y}=\left(\Pi_{2}+\Sigma_{Y}\right), \\
& B=-A^{X} H+B^{X}, \quad B^{\prime}=-A^{Y} H+B^{Y}, \\
& R(Z)=\frac{\left(A^{X}\right)^{2}+\left(A^{Y}\right)^{2}}{A^{X} B^{\prime}-A^{Y} B} Z+\frac{A^{X} B+A^{Y} B^{\prime}}{A^{X} B^{\prime}-A^{Y} B}, \\
& R_{0}=\frac{A^{X} B+A^{Y} B^{\prime}}{A^{X} B^{\prime}-A^{Y} B} .
\end{aligned}
$$

Also, ${ }_{2} F_{1}$ stands for hypergeometric function and is defined by power series as:

$$
{ }_{2} F_{1}(a, b ; c ; z)=\sum_{j=0}^{\infty} \frac{(a)_{j}(b)_{j}}{(c)_{j}} \frac{z^{j}}{j !} .
$$

By integrating Eqs. (26a) and (26b) over the film thickness, and substituting the results into Eq. (24), the evolution equation in three-dimensional form is achieved. Integrating the velocity components, $U$ and $V$ over the film thickness can be problematic and mathematically difficult to perform. Therefore, in order to simplify Eqs. (26a) and (26b) and make them easier to integrate, first, the power-law index based on the liquid rheology is determined. Then, by substituting the results into Eq. (24), the governing evolution equation is obtained. For example, for $n=$ $1 / 3$, the evolution equation for a three-dimensional case is:

$$
\begin{aligned}
H_{T} & +\frac{\partial}{\partial X}\left\{\frac{A^{X}}{20}\left(\left(A^{X}\right)^{2}+\left(A^{Y}\right)^{2}\right) H^{5}\right. \\
& +\frac{1}{4}\left(\frac{2}{3} A^{Y}\left(A^{Y} B-A^{X} B^{\prime}\right)+B\left(\left(A^{X}\right)^{2}+\left(A^{Y}\right)^{2}\right)\right) H^{4} \\
& +\frac{1}{3}\left(\frac{1}{2} A^{X}\left(\left(B^{\prime}\right)^{2}+B^{2}\right)+B\left(A^{Y} B^{\prime}+A^{X} B\right)\right) H^{3} \\
& \left.+\frac{1}{2} B\left(B^{2}+\left(B^{\prime}\right)^{2}\right) H^{2}\right\} \\
& +\frac{\partial}{\partial Y}\left\{\frac{A^{Y}}{20}\left(\left(A^{X}\right)^{2}+\left(A^{Y}\right)^{2}\right) H^{5}\right. \\
& +\frac{1}{4}\left(\frac{2}{3} A^{X}\left(A^{Y} B^{-} A^{X} B^{\prime}\right)+B^{\prime}\left(\left(A^{X}\right)^{2}+\left(A^{Y}\right)^{2}\right)\right) H^{4} \\
& +\frac{1}{3}\left(\frac{1}{2} A^{Y}\left(\left(B^{\prime}\right)^{2}+B^{2}\right)+B^{\prime}\left(A^{Y} B^{\prime}+A^{X} B\right)\right) H^{3} \\
& \left.+\frac{1}{2} B^{\prime}\left(\left(B^{\prime}\right)^{2}+B^{2}\right) H^{2}\right\}=0 .
\end{aligned}
$$

Similarly, for two-dimensional cases, we have Eq. (30) as shown in Box III. Eq. (30) expresses the evolution of thin films for a non-Newtonian fluid. In this equation, external forces, conservative body forces, and surface tension effects are considered. This equation and that

$$
\begin{aligned}
& P=-\Pi_{3}-\beta H_{X X} \\
& U(Z)=\frac{n\left(\left(\left|(Z-H) A^{X}+B^{X}\right|\right)^{1+1 / n}-\left(\left|-A^{X} H+B^{X}\right|\right)^{1+1 / n}\right)}{A^{X}(n+1)} \\
& H_{T}+\frac{n^{2}}{(n+1)(2 n+1)} \frac{\partial}{\partial X}\left[\frac{B^{X}\left(\left|B^{X}\right|\right)^{1+1 / n}}{\left(A^{X}\right)^{2}}-\frac{\left(B^{X}+\frac{n+1}{n} A^{X} H\right)}{\left(A^{X}\right)^{2}}\left(\left|B^{X}-A^{X} H\right|\right)^{1+1 / n}\right]=0 .
\end{aligned}
$$


of the Newtonian one will converge if the power-law index is equal to $1 . n>1$ refers to dilatant behavior and $n<1$ refers to pseudo plastic behavior.

\subsection{Effects of gravity and constant surface tension forces on thin films}

As a very simple case, a thin film of fluid, which is on the top of a horizontal solid surface and under the influence of gravity and surface tension forces, is considered. Then, parameters $A^{X}$ and $B^{X}$ are determined by:

$$
\begin{aligned}
A^{X} & =(P+\Phi)_{X}=\left(-\Pi_{3}-\beta H_{X X}+G(H-Z)\right)_{X} \\
& =G H_{X}-\beta H_{X X X}, \\
B^{X} & =\left(\Pi_{1}+\Sigma_{X}\right)=0,
\end{aligned}
$$

where, $G=\rho g d^{2} / \eta_{0} U_{0}$ is the gravity number. Substituting the above equations into Eq. (30c), we have:

$$
\begin{array}{r}
H_{T}-\frac{n}{(2 n+1)}\left[H^{2}\left(G H H_{X}-\beta H H_{X X X}\right)\right. \\
\left.\left(\left|G H H_{X}-\beta H H_{X X X}\right|\right)^{\frac{1-n}{n}}\right]_{X}=0 .
\end{array}
$$

The dimensional version of Eq. (32) is:

$$
\begin{array}{r}
K^{1 / n} h_{t}-\frac{n}{(2 n+1)}\left[h^{2}\left(\rho g h h_{x}-\sigma h h_{x x x}\right)\right. \\
\left.\left(\left|\rho g h h_{x}-\sigma h h_{x x x}\right|\right)^{\frac{1-n}{n}}\right]_{x}=0 .
\end{array}
$$

To investigate instability properties of a film perturbed by a small disturbance, the linear-stability of Eq. (32) is studied. A uniform film, $H=\bar{H}$, perturbed by a small disturbance, $H^{\prime}\left(H^{\prime} \ll 1\right)$, is considered. Since the coefficients in the equations are independent of time and $X$, the separable solution to the form $H^{\prime}=$ $H_{0}^{\prime} e^{(i k X+\omega T)}, H_{0}^{\prime}=$ cost. is sought, where $k$ is the wave number in $X$-direction. Substituting them into Eq. (32) and linearizing it with respect to $H^{\prime}, \omega$ is obtained as:

$$
\omega=\frac{-n k^{2} \bar{H}}{2 n+1}\left(G \bar{H}^{2}+\bar{H}^{2} k^{2} \beta\right) .
$$

The sign $\operatorname{Re}(\omega)$ corresponds to the variation of the perturbation amplitude and pure imaginary value of $\omega$ corresponds to wave translation along $X$-axis. If the real part of $\omega$ is negative, the amplitude of perturbation will decay while it will grow if $\operatorname{Re}(\omega)$ is positive.

In the cases that liquid film is positioned under a plate, the sign of $G$ in Eq. (34) changes and it is replaced by $-G$. This case is known in the literature as the Rayleigh-Taylor instability of a thin viscous layer. In such situation, the Bond number, which is defined

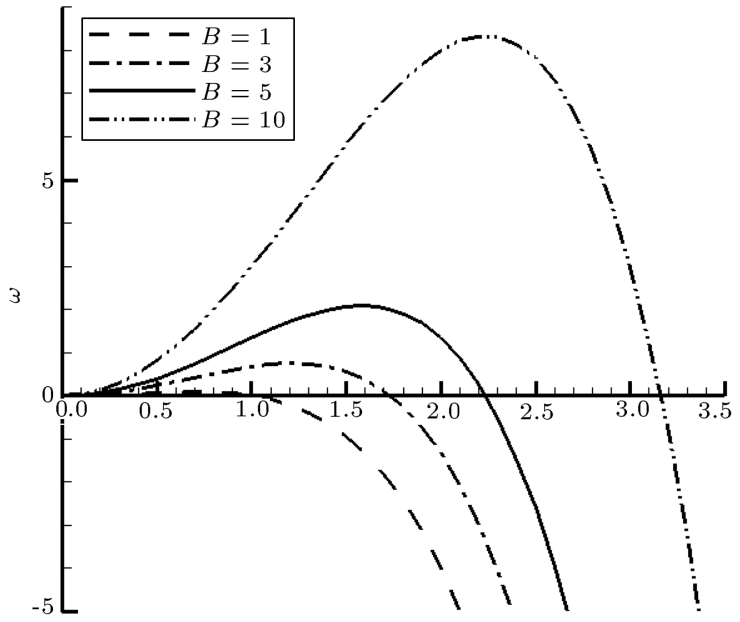

Figure 2. Variation of $\omega$ versus non-dimensional wavenumber $k$ for various values of $\beta$.

as the ratio of gravitational to surface tension forces, is:

$$
\text { Bond number }=\frac{\rho g d^{2}}{\sigma},
$$

which is proportional to $B=G / \beta$ and plays an important role in stability of the film. Figure 2 shows variation of $\omega$ versus the dimensionless wavenumber $k$ for $B=1,3,5$, and 10 . It is seen that the cutoff wavenumber for neutral stability increases with the Bond number. Also, there is a maximum point in each graph, indicating the wavenumber at which the perturbation leads to the most unstable film.

\subsection{Numerical solution}

It is showed that the evolution of a thin film for power-law liquid under the act of gravity and constant surface tension forces is governed by Eq. (32). Here, this equation is solved numerically for some sample cases including a uniform thin film perturbed by a disturbance on its surface in presence of the RayleighTaylor instability.

\subsubsection{Numerical method}

Eq. (32) is first order in time and fourth order in space and is a highly non-linear differential equation. In order to numerically solve this equation, the fourth order Runge-Kutta method for time derivative and second order central difference for space derivatives have been used.

\subsubsection{Grid study}

In order to show the independence of the solutions from the time step and grid size, Eq. (32) is solved numerically for $G=-1, \beta=1, n=1.0$, and initial condition of $H_{\text {ini. }}=1.0+0.1 \cos (X / \sqrt{2})$. First, the time step for grid size of 100 is examined. Figure 3(a) shows that for $\Delta T \leq 2.0 \times 10^{-6}$, the results are independent of $\Delta T$.

The numerical solution is repeated for grid sizes 


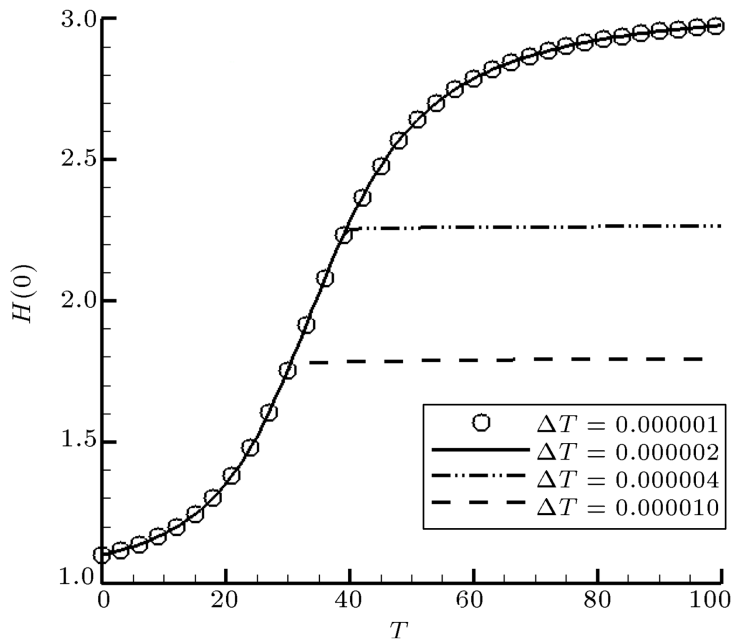

(a)

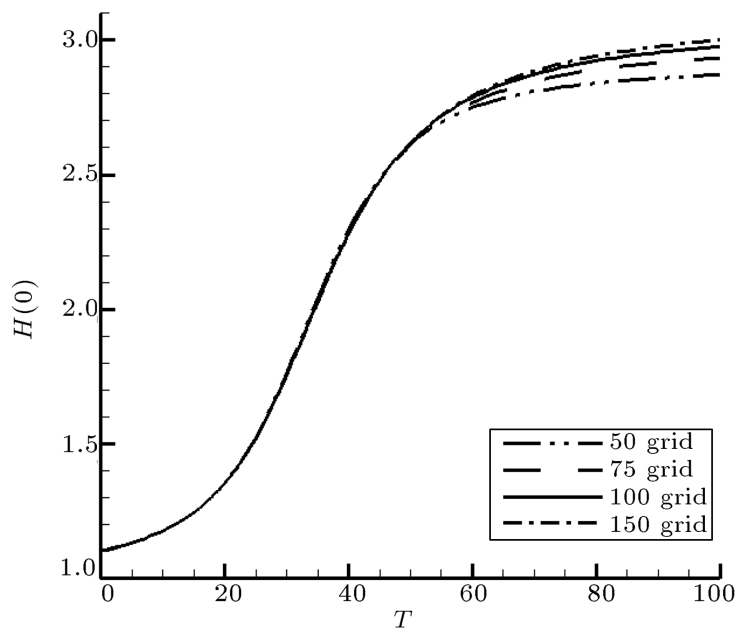

(b)

Figure 3. (a) Variation of $H(0)$ as a function of time for grid size of 100 and different time steps. (b) Variation of $H(0)$ as a function of time for $\Delta T=2.0 \times 10^{-6}$ and different grid sizes.

associated with 75, 100, and 150 cells along one wavelength for $\Delta T=2.0 \times 10^{-6}$. According to Figure 3(b), the grid size associated with 100 cells $(\Delta X \cong 0.18)$ and time step $\Delta T=2.0 \times 10^{-6}$ is appropriate and chosen.

\subsubsection{Validation}

In order to validate the results, the result of the Rayleigh-Taylor instability of a thin film for Newtonian liquid obtained by Yiantsios and Higgins [3] is reproduced. The initial disturbance on the film surface is $H_{\text {ini. }}=1.0+0.1 \cos (X / \sqrt{2})$. Eq. (32) is numerically solved by considering $n=1$. Figure 4 shows the present numerical solution result and that of [3]. As can be seen, these results are in very good agreement.

\section{Results and discussion}

Considering an element of fluid positioned on the film surface with mean depth of $d$, its gravitational force is

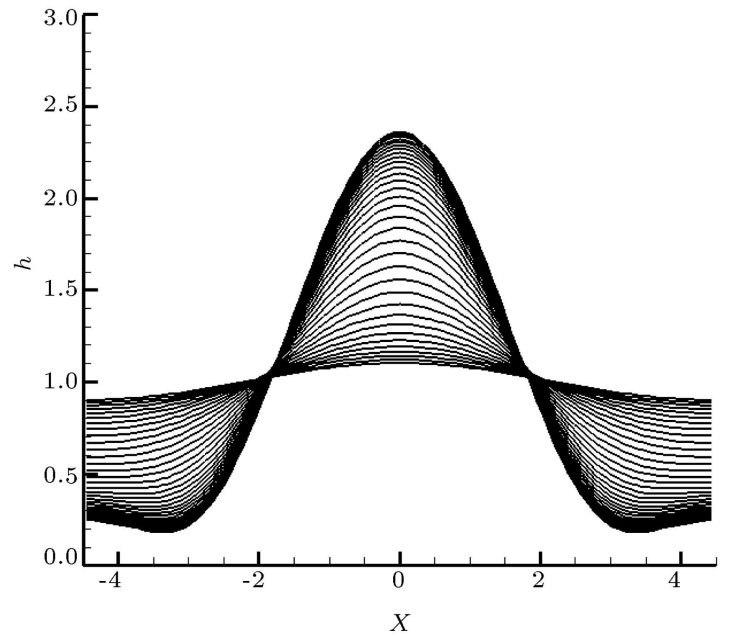

(a)

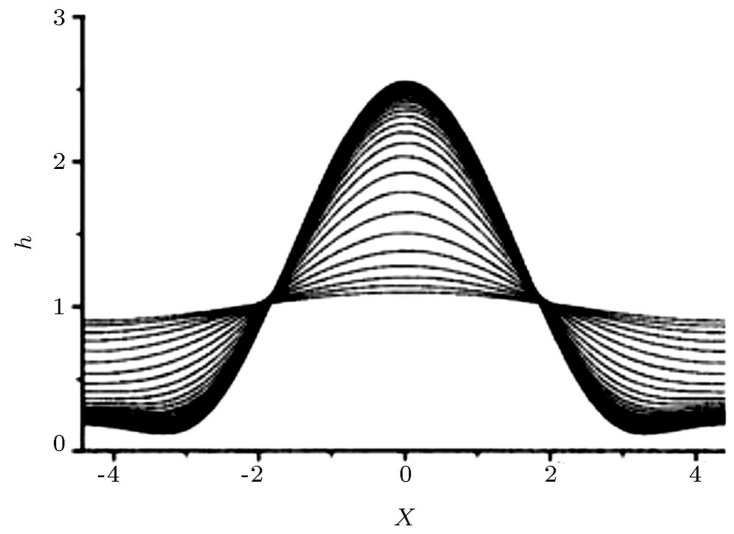

(b)

Figure 4. Free surface of a thin film for $G=-1, \beta=1$, and $n=1.0$ at different times, time intervals $\Delta t=4 \mathrm{~s}$ and maximum time $t_{\max }=100 \mathrm{~s}$ : (a) Present results and (b) Yiantsios and Higgins [3].

$\rho g d$. Let us consider $L$ as the length scale parallel to the film surface; then, $\sigma d / L^{2}$ is a measure of interfacial force. When the film is on a solid plate, these two forces act to stabilize the film. On the other hand, if the film is positioned under the solid plate, the gravitational force acts as a source of instability, while interfacial force stabilizes the flow. In such system, the balance of these two forces gives a cutoff wavelength. The disturbed films remain stable if the wavelength is smaller than the cutoff value. For thin films, $d \ll L$, this analysis requires Bond number $\approx d^{2} / L^{2}=\varepsilon^{2} \ll 1$. Therefore, small Bond number refers to the situation in which the surface tension force is balanced by the gravity force.

\subsection{Evolution of thin films perturbed by a periodic disturbance for $G>0$}

The initial condition is taken to be $H_{\text {ini. }}=1.0+A$ $\cos (X / \sqrt{2})$, where $A$ is the wave amplitude and is constant. Figure 5 presents free surface profiles of the thin films at $T=10$ for pseudo plastic, dilatant, and Newtonian fluids. Since small Bond number is 


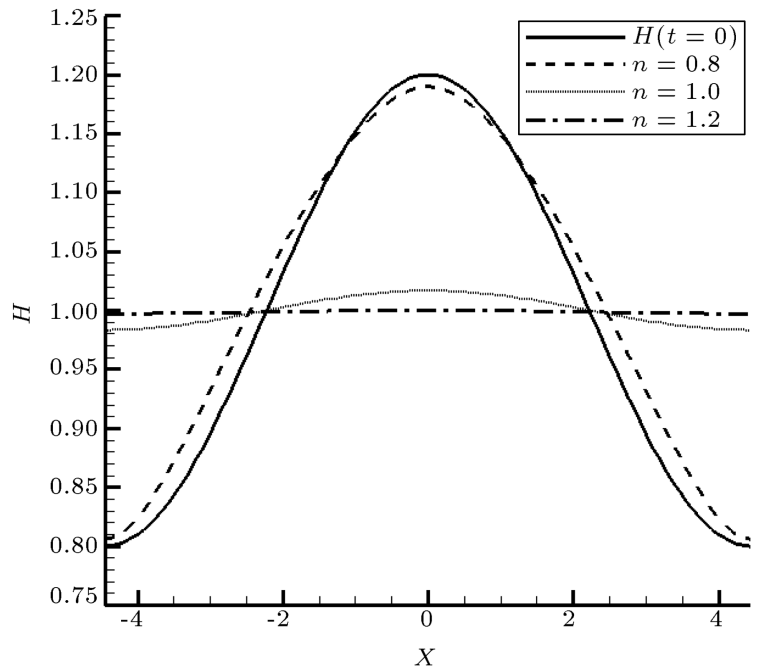

Figure 5. Free surface profile at $T=10$ for $G=1, \beta=1$, and $A=0.2$.

considered, the parameters $G, \beta$, and $A$ are chosen to be 1,1 , and 0.2 , respectively. As it is seen, the gravitational and interfacial forces damp the disturbance. The point is that in the pseudo plastic, the required time for damping the disturbance is much larger than that in the Newtonian and dilatant fluids; and dilatant behavior is damped faster than in the Newtonian fluids. It is due to the fact that the fluid velocity and its viscosity coefficient are obtained from Eqs. (30b) and (25), respectively, and, based on those equations, for the same free surface profile, the pseudo plastic fluid has lower velocity engaged with much larger viscosity. Therefore, pseudo plastic fluid needs more time to damp the perturbation.

Figure 6 shows the free surface evolution for a larger Bond number. These results are obtained for $G=500, \beta=1$, and $A=0.5$. It is shown that in those cases, the disturbance is damped faster due to increase in the gravity force. It should be mentioned that the invalidity of power-law model for low strain rate causes inaccurate prediction of free surface evolution for pseudo plastic fluids, as it is shown in Figure 6(a). Unphysical sharp changes of the curves at low and high $X$ 's are due to the behavior of the power-law model for pseudo plastic fluids $(n<1)$.

The evolution of the free surface for different wave amplitudes, $A=0.5,0.2$, and 0.1 , and different wavenumbers, $k=\sqrt{2} / 2, \sqrt{2}$, and $3 \sqrt{2} / 2$, is studied. In all these cases, the results show similar trends in free surface evolutions. The only difference here is the damping time. As it can be concluded from linearstability of the problem (Eq. (34)), the perturbing waves with larger wavenumbers change the film free surface shape much faster.

\subsection{Rayleigh-Taylor instability}

The Rayleigh-Taylor instability for power-law nonNewtonian fluids is investigated and the results are compared with those for Newtonian fluids. For this purpose, the initial perturbing disturbance on the film surface is assumed $H_{\text {ini. }}=1.0+0.1 \cos (k X)$, where the wavenumber $k$ is $\sqrt{2} / 2$. The reason for choosing $\sqrt{2} / 2$ is that for this value of $k$, the sinusoidal disturbances have maximum growth rate according to the linear stability theory, as it can be verified by Eq. (34). Figure 7 illustrates the evolutions of four different fluids for $G=-1, \beta=1$, and $k=\sqrt{2} / 2$. It is seen that for all the four cases, the film evolutions experience three different stages. First, for a very short time, the evolution follows the linear stability prediction. Then, the non-linearity causes generation of higher harmonics and one drop per wavelength is formed. Furthermore, as it can be seen from Eq. (32), where the driving force is multiplied by $H^{3}$, the crests of the waves become more noticeable than their troughs. In other words, where the film is thinner, it is harder to flow due to no slip boundary condition at the solid surface. Finally, as time progresses, the film evolution slows down and eventually reaches the steady-state form. From the

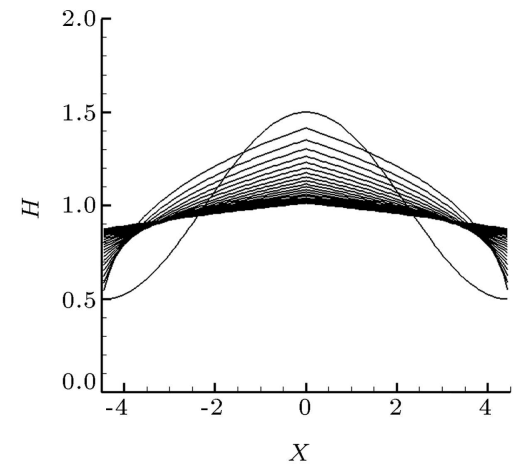

(a)

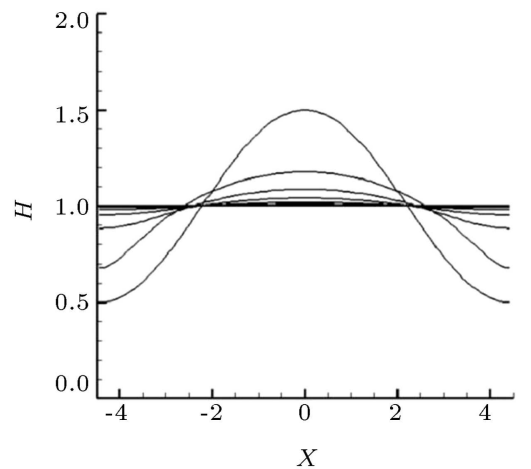

(b)

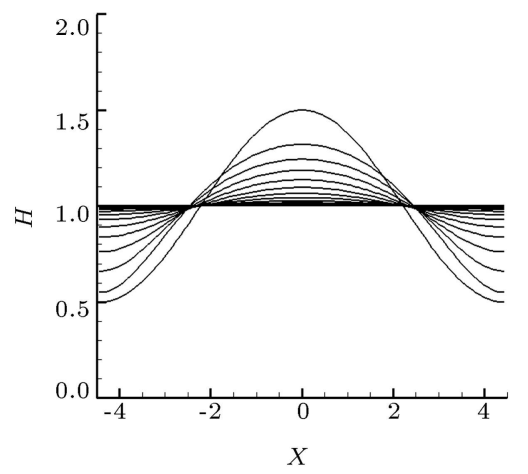

(c)

Figure 6. Evolution of free surface for $G=500, \beta=1$, and $A=0.5$ : (a) $n=0.8$, time interval, $\Delta T$, is 0.1 , maximum time, $T_{\max }$ is 2 ; (b) $n=1.0$, time interval, $\Delta T$, is 0.01 , maximum time, $T_{\max }$, is 0.1 , and (c) $n=1.2$, time interval, $\Delta T$, is 0.01 , maximum time, $T_{\max }$, is 0.1 . 


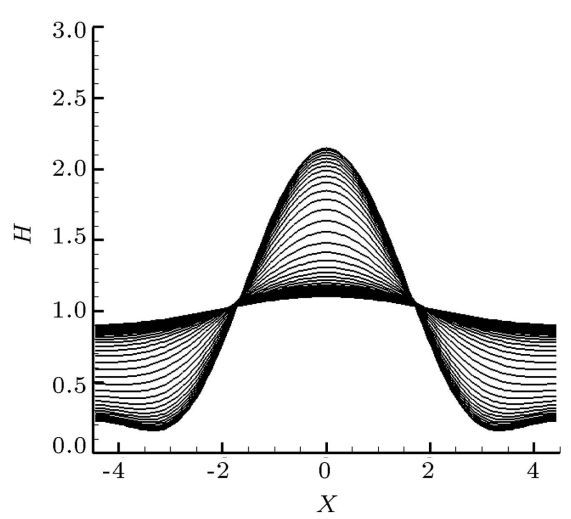

(a)

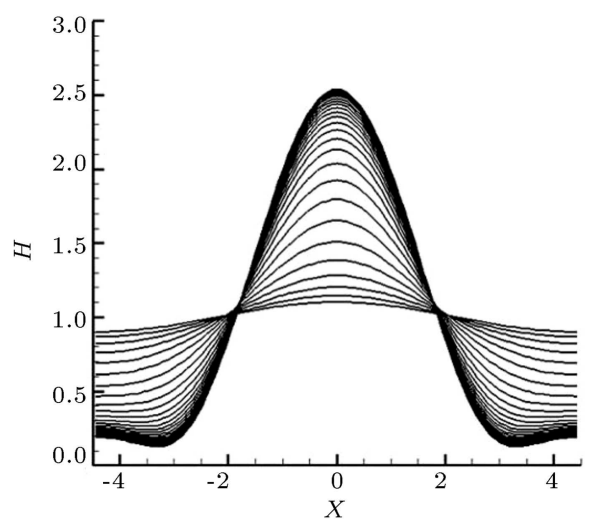

(c)

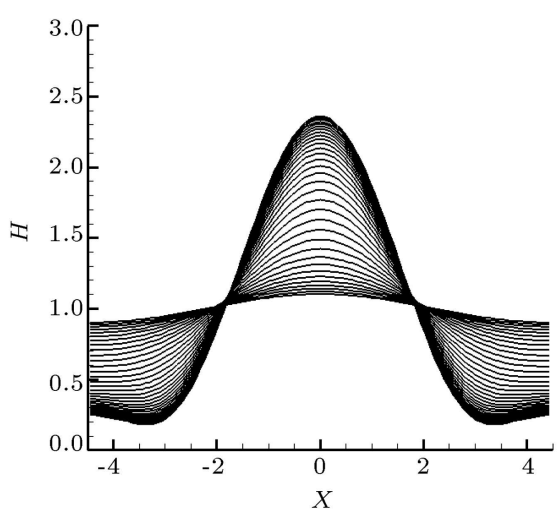

(b)

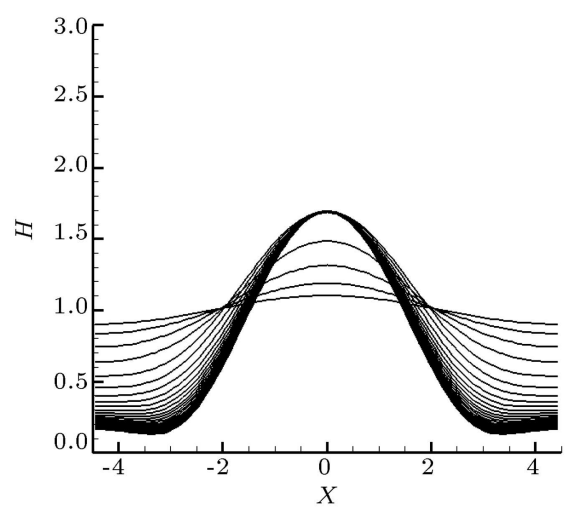

(d)

Figure 7. Evolution of free surface for $G=-1, \beta=1$, and $k=22$ : (a) $n=0.8$, time interval, $\Delta T$, is 16 , maximum time, $T_{\max }$, is 640 , (b) $n=0.9$, time interval, $\Delta T$, is 8 , maximum time, $T_{\max }$, is 132 , (c) $n=1.0$, time interval, $\Delta T$, is 4 , maximum time, $T_{\max }$, is 100 , and (d) $n=1.2$, time interval, $\Delta T$, is 4 , maximum time, $T_{\max }$, is 100 .

numerical results, it is concluded that rheology of the fluid does not affect the trend of evolution of the film; it only influences the required time to progress and affects the amplitude of the final shape. As it is demonstrated in Figure 7, the pseudo plastic fluids flow is slower than Newtonian and dilatant fluids flows. This agrees with the results obtained by Houseman and Molnar [8] that the growth of instability strongly depends on the constitutive law relating stress and strain rate such that instability grows exponentially for Newtonian fluids and super exponentially for non-Newtonian ones. Also, the form of the free surface shape in their evolution is similar to those in the results of $[6,8]$.

In order to understand the effect of wavenumber on evolution of film surface, $\sqrt{2}$ and $3 \sqrt{2} / 2$ are taken as initial perturbing disturbance wavenumbers, too. From the results, it is observed that for all pseudo plastic, Newtonian, and dilatant fluids, two and three drops per wavelength have been formed for $k=\sqrt{2}$ and $3 \sqrt{2} / 2$, respectively. This has been also shown by Yiantsios and Higgins [3] for Newtonian fluids. Here, similar trends for pseudo plastic and dilatant fluids are observed. Thus, the fluid rheology does not have effect on this trend.

In Figure 8, the Bond number increases by in- crease in the value of gravity number, $G$. These results, which are achieved for $G=-10, \beta=1$, and $k=\sqrt{2} / 2$, show the evolution of pseudo plastic, Newtonian, and dilatant fluids, respectively. Here, again, the thin films experience the same three stages during their development. As it is shown in Figure 8, the numbers of drops formed per wavelength for pseudo plastic, Newtonian, and dilatant fluids are two, three, and three, respectively. Moreover, the trend of pseudo plastic fluids evolution toward the final shape differs from those of Newtonian and dilatant fluids. Since the increase in the value of Bond number causes more instability, which also transfers the problem to a higher non-linear one, the transition near the inflection points is more pronounced in this case for the smaller Bond numbers (Figure 7).

The Rayleigh-Taylor instability is studied for larger values of Bond number. From the results, it can be seen that at first the film evolves gradually with time; then, higher harmonics start to grow and this speeds up. In these cases, the gravity force is much stronger than the interfacial force and the film becomes unstable. Therefore, in the first iterations, the film evolution is captured; but, after a short time, its transition speeds up and the value of the 


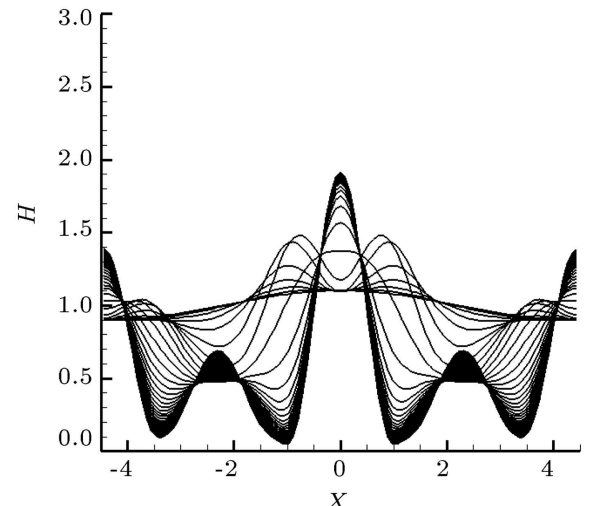

(a)

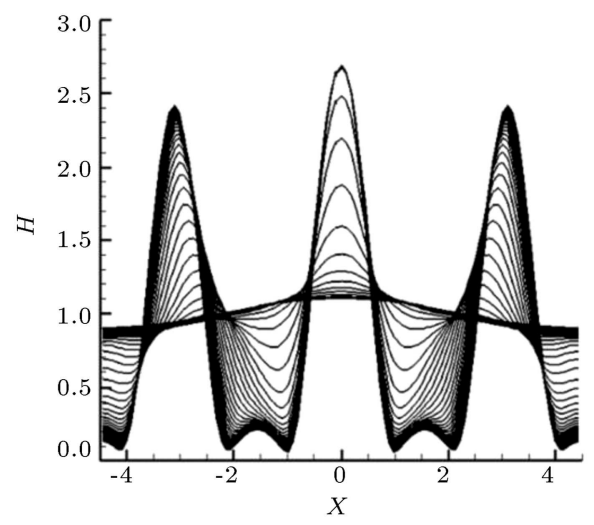

(c)

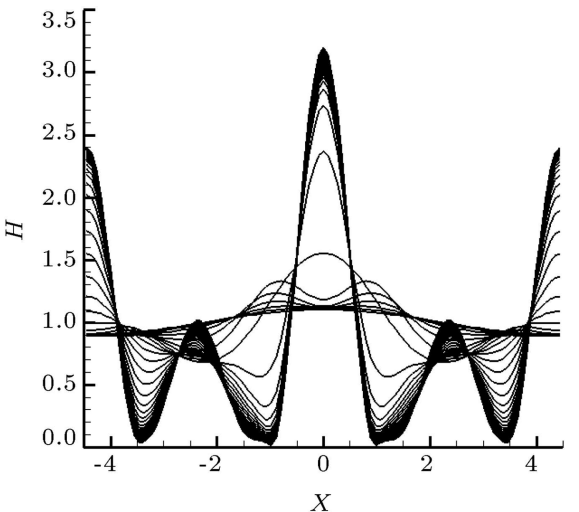

(b)

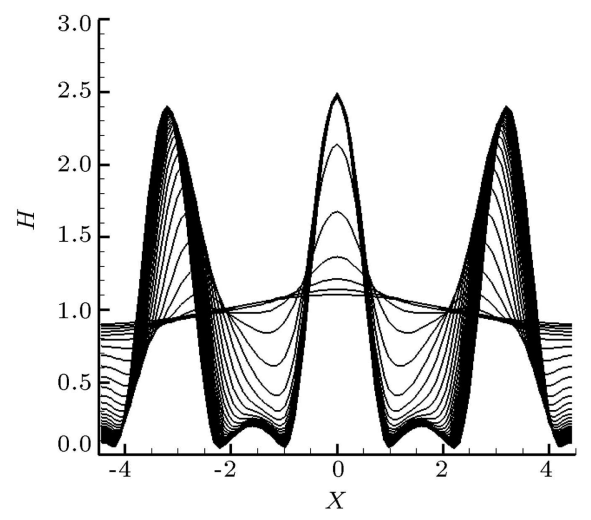

(d)

Figure 8. Evolution of free surface for $G=-10, \beta=1$, and $k=22$ : (a) $n=0.8$, time interval, $\Delta T$, is 0.1 , maximum time, $T_{\max }$, is 4 , (b) $n=0.9$, time interval, $\Delta T$, is 0.1 , maximum time, $T_{\max }$, is 4 , (c) $n=1.0$, time interval, $\Delta T$, is 0.05 , maximum time, $T_{\max }$, is 2.5 , and $(\mathrm{d}) n=1.2$, time interval, $\Delta T$, is 0.1 , maximum time, $T_{\max }$, is 4 .

film dimensionless thickness, $H$, tends to infinity. As Fermigier et al. [6] have shown, this is the time when a drop separates from the film. Their results showed that there was a critical Bond number above which the drops spontaneously fell and below which the film remained stable. Our method for solving the problem does not have the ability to capture the drop and its trend of creation.

\section{Conclusion}

The governing equations of a power law non-Newtonian liquid film on a solid plane have been derived within the lubrication approximation. The possibility of the inclusion of interfacial forces as well as presence of conservative forces, such as gravity, centrifugal, or van der Waals, and surface tension has been considered. The governing system of equations is solved and highly non-linear differential equations for two- and threedimensional cases, which describe the evolution of the thin films well, are presented. These equations can be used as a starting point to investigate the influence of rheology on the evolution of thin film for nonNewtonian fluids.

The evolution of thin films is studied when the film is below a solid surface and subjected to the influence of gravity and constant surface tension forces. The stability properties of a uniform film perturbed by a small periodic disturbance are studied and the evolution equation of thin film has been solved numerically in order to compare the behavior of Newtonian and non-Newtonian liquid films for different Bond numbers. It is shown that due to the invalidity of power-law model at low and high strain rates, the results do not have a reasonable and physical meaning. Also, it is concluded that dilatant fluids respond to the disturbance more quickly than Newtonian and pseudo plastic fluids do. The Rayleigh-Taylor instability is also studied and the behaviors of pseudo plastic, dilatant, and Newtonian fluids are compared. The results show that the trend of evolution of free surface for pseudo plastic fluids is different from that for Newtonian and dilatant fluids. For small Bond numbers, the thin film experiences three stages as it evolves:

1. Within a very short time, it follows the linear stability prediction;

2. The non-linearity effects cause generation of higher harmonics and drops are formed; 
3. The transition slows down and a steady-state shape forms the film surface.

It is shown that by increasing the Bond number, the non-linearity effect would be more pronounced. In this situation, the imbalance between the gravitational and surface tension forces makes the film unstable and drops are created. The method used here is not capable of capturing the separated drops and the trend of their formation.

\section{Nomenclature}

A Wave amplitude

$A^{X}, A^{Y} \quad$ Coefficient in Eq. (28)

$B, B^{\prime} \quad$ Coefficient in Eq. (28)

$B^{X}, B^{y} \quad$ Coefficient in Eq. (28)

$\mathrm{Ca}^{-1} \quad$ Inverse of the capillary number

$d \quad$ Mean film thickness

$D \quad$ Strain rate tensor

$g \quad$ Gravitational acceleration

G Gravity number

$h \quad$ Dimensional film thickness

$H \quad$ Dimensionless film thickness

$\widetilde{H} \quad$ Mean curvature of the film surface

$k \quad$ Wave number

$K \quad$ Constant in Eq. (5)

$L \quad$ Length scale

$n \quad$ Power-law index

$p \quad$ Pressure

$P \quad$ Dimensionless pressure

Re Reynolds number

$s \quad$ Strain rate

$S \quad$ Dimensionless strain rate

$T \quad$ Dimensionless time

$u \quad$ Velocity in $x$-direction

$U \quad$ Dimensionless velocity in $X$-direction

$v \quad$ Velocity in $y$-direction

$V \quad$ Dimensionless velocity in $Y$-direction

$w \quad$ Velocity in $z$-direction

$W \quad$ Dimensionless velocity in $Z$-direction

${ }_{2} F_{1} \quad$ Hyper geometric function

$\sim \quad$ Approximately

\section{Greek symbols}

$\Pi \quad$ Dimensionless interfacial stress

$\Sigma \quad$ Dimensionless surface tension

$\Phi \quad$ Dimensionless body force potential

$\Lambda \quad$ Dimensionless viscosity $\beta$

$\varepsilon$

$\eta$

$\lambda$

$\mu$

$\pi$

$\rho$

\section{$\sigma$}

$\tau$

$\omega$

$\varphi$

\section{Subscripts}

$\begin{array}{ll}T, t & \text { Derivatives on } T \text { and } t \\ X, x & \text { Derivatives on } X \text { and } x \\ Y, y & \text { Derivatives on } Y \text { and } y \\ Z, z & \text { Derivatives on } Z \text { and } z \\ 0 & \text { Characteristic values } \\ 1 & \text { Normal to the film surface } \\ 2 & \text { Tangential to the film surface } \\ 3 & \text { Tangential to the film surface } \\ \max & \text { Maximum } \\ \text { ini. } & \text { Initial value }\end{array}$

\section{References}

1. Gad-el-Hak, M., The MEMS Handbook, CRC Press (2001).

2. Oron, A., Davis, S.H. and Bankoff, S.G. "Long-scale evolution of thin liquid films", Rev. Modern Phys., 68, pp. 931-980 (1997).

3. Yiantsios, S.G. and Higgins, B.G. "Rayleigh-Taylor instability in thin viscous films", Phys. Fluids, 1, pp. 1484-1501 (1989).

4. Yiantsios, S.G. and Higgins, B.G. "Rapture of thin films: nonlinear stability analysis", J. Colloid Interface Sci., 147, pp. 341-350 (1991).

5. Oron, A. and Rosenau, P. "Formation of patterns induced by thermocapillarity and gravity", J. Phy. (France), 2, pp. 131-146 (1992).

6. Fermigier, M., Limat, L., Wesfreid, J.E., Boudient, P. and Quilliet, C. "Two-dimensional patterns in Rayleigh-Taylor instability of a thin layer", J. Fluid Mech., 236, pp. 349-383 (1992).

7. Ruyer-Quil, C. and Manneville, P. "Improved modeling of flows down inclined planes", Eur. Phys. J. B, 15, pp. 357-369 (2000).

8. Houseman, G.A. and Molnar, P. "Gravitational (Rayleigh-Taylor) instability of a layer with non-linear viscosity and convective thinning of continental lithosphere", Int. J. Geophys., 128, pp. 125-150 (1997). 
9. Fomin, S., Watterson, J. and Raghunathan, S. "The run-off condition for rimming flow of a power-law fluid", Theoret. Comput. Fluid Dynamics, 15, pp. 8394 (2001).

10. Perazzo, C.A. and Gratton, J. "Thin film of nonNewtonian fluid on an incline", Phusical Review, 67, pp. 1-6 (2003).

11. Perazzo, C.A. and Gratton, J. "Steady and traveling flows of a power-law liquid over an incline", J. NonNewtonian Fluid Mech., 118, pp. 57-64 (2004).

12. Balmforth, N., Ghadge, Sh. and Myers, T. "Surface tension driven fingering of a viscoplastic film", J. NonNewtonian Fluid Mech., 142, pp. 143-149 (2007).

13. Miladinova, S., Lebon, G. and Toshev, E. "Thin-film flow of a power-law liquid falling down an inclined plate", J. Non-Newtonian Fluid Mech., 122, pp. 6978 (2004).

14. Myers, T.G. "Application of non-Newtonian models to thin film flow", Physical Review, 72, pp. 1-11 (2005).

15. Perazzo, C.A. and Gratton, J. "Exact solutions for two-dimensional steady flows of a power-law liquid on an incline", Phys. Fluids, 17, pp. 1-8 (2005).

16. Heining, C. and Aksel, N. "Effects of inertia and surface tension on a power-law fluid flowing down a wavy incline", Int. J. Multiphase Flow, 36, pp. 847857 (2010).

17. Hu, B. and Kieweg, S.L. "The effect of surface tension on the gravity-driven thin film flow of Newtonian and power-law fluids", Computers \& Fluids, 64, pp. 83-90 (2012).

\section{Appendix}

By solving Eqs. (23) for $U_{Z}$ and $V_{Z}$ and substituting Eq. (27) into its results, we will have a system of two non-linear equations as:

$$
\begin{aligned}
& U_{Z}=\frac{(Z-H) A^{X}+B^{X}}{\left[U_{Z}^{2}+V_{Z}^{2}\right]^{(n-1) / 2}}, \\
& V_{Z}=\frac{(Z-H) A^{Y}+B^{Y}}{\left[U_{Z}^{2}+V_{Z}^{2}\right]^{(n-1) / 2}} .
\end{aligned}
$$

By solving Eqs. (A.1) and (A.2), $U_{Z}$ and $V_{Z}$ are obtained:

$$
\begin{aligned}
& U_{Z}(Z) \\
& =\frac{(Z-H) A^{X}+B^{X}}{\left[\left((Z-H) A^{X}+B^{X}\right)^{2}+\left((Z-H) A^{Y}+B^{Y}\right)^{2}\right]^{(n-1) / 2 n}},
\end{aligned}
$$

$V_{Z}(Z)$

$$
=\frac{(Z-H) A^{Y}+B^{Y}}{\left[\left((Z-H) A^{X}+B^{X}\right)^{2}+\left((Z-H) A^{Y}+B^{Y}\right)^{2}\right]^{(n-1) / 2 n}} .
$$

To obtain $U(Z)$ and $V(Z)$, Eqs. (A.3) and (A.4) are integrated over the film thickness from 0 to $Z$. Here, the results are shown for $U(Z)$; Similar procedure can be followed to obtain $V(Z)$ :

$$
\begin{aligned}
U(Z) & =\int_{0}^{Z} U_{\xi}(\xi) d \xi \\
= & \int_{0}^{Z} \frac{(\xi-H) A^{X}+B^{X}}{\left[\left((\xi-H) A^{X}+B^{X}\right)^{2}+\left((\xi-H) A^{Y}+B^{Y}\right)^{2}\right]^{(n-1) / 2 n}} d \xi .
\end{aligned}
$$

Using $G(Z)$ and $L(Z)$ to rewrite Eq. (A.5) in a way that $U(Z)=Q(Z)+E(Z)$, we have:

$$
\begin{aligned}
Q(Z)= & \frac{A^{X}}{2\left[\left(A^{X}\right)^{2}+\left(A^{Y}\right)^{2}\right]} \\
& \int_{0}^{Z} \frac{2\left[\left(A^{X}\right)^{2}+\left(A^{Y}\right)^{2}\right] \xi+2\left(A^{X} B+A^{Y} B^{\prime}\right)}{\left[\left(A^{X} \xi+B\right)^{2}+\left(A^{Y} \xi+B^{\prime}\right)^{2}\right]^{(n-1) / 2 n}} d \xi, \\
E(Z)= & \left(B-\frac{A^{X}\left(A^{X} B+A^{Y} B^{\prime}\right)}{\left(A^{X}\right)^{2}+\left(A^{Y}\right)^{2}}\right) \\
& \int_{0}^{Z} \frac{1}{\left[\left(A^{X} \xi+B\right)^{2}+\left(A^{Y} \xi+B^{\prime}\right)^{2}\right]^{(n-1) / 2 n}} d \xi,
\end{aligned}
$$

where $Q(Z)$ is the integral of a function, which is easily calculated by the general rule; for $f(x)=x^{q}$ with $q \neq-1$, the anti-derivative is $F(x)=x^{q+1} /(q+1)$. Therefore, we have:

$$
\begin{aligned}
Q(Z) & =\frac{A^{X}}{\left(A^{X}\right)^{2}+\left(A^{Y}\right)^{2}} \frac{n}{n+1} \\
& \left\{\left[\left(A^{X} Z+B\right)^{2}+\left(A^{Y} Z+B^{\prime}\right)^{2}\right]^{(n+1) / 2 n}\right. \\
- & {\left.\left[(B)^{2}+\left(B^{\prime}\right)^{2}\right]^{(n+1) / 2 n}\right\} . }
\end{aligned}
$$

To calculate the integral part of Eq. (A.7), $R(\xi)$ is defined as:

$$
\begin{aligned}
& R(\xi)=\frac{\left(A^{X}\right)^{2}+\left(A^{Y}\right)^{2}}{A^{X} B^{\prime}-A^{Y} B} \xi+\frac{A^{X} B+A^{Y} B^{\prime}}{A^{X} B^{\prime}-A^{Y} B}, \\
& d R=\frac{\left(A^{X}\right)^{2}+\left(A^{Y}\right)^{2}}{A^{X} B^{\prime}-A^{Y} B} d \xi .
\end{aligned}
$$

Substituting Eq. (A9) into Eq. (A7), we have:

$$
\begin{gathered}
E(Z)=\frac{-A^{Y}\left(\left|A^{X} B^{\prime}-A^{Y} B\right|\right)^{(n+1) / n}}{\left[\left(A^{X}\right)^{2}+\left(A^{Y}\right)^{2}\right]^{(3 n+1) / 2 n}} \\
\int_{R_{0}}^{R(Z)} \frac{1}{\left(R^{2}+1\right)^{(n-1) / 2 n}} d R,
\end{gathered}
$$

where, $R_{0}=\left(A^{X} B+A^{Y} B^{\prime}\right) /\left(A^{X} B^{\prime}-A^{Y} B\right)$. The hy- 
pergeometric function ${ }_{2} F_{1}(a, b ; c ; \xi)=\sum_{j=0}^{\infty} \frac{(a)_{j}(b)_{j}}{(c)_{j}} \frac{\xi^{j}}{j !}$ is used to calculate the integral part of Eq. (A.10). Therefore, $E(Z)$ is:

$$
\begin{aligned}
E(Z)= & \frac{-A^{Y}\left(\left|A^{X} B^{\prime}-A^{Y} B\right|\right)^{(n+1) / n}}{\left[\left(A^{X}\right)^{2}+\left(A^{Y}\right)^{2}\right]^{(3 n+1) / 2 n}} \\
& \left\{R(Z)_{2} F_{1}\left(\frac{1}{2}, \frac{-1+n}{2 n} ; \frac{3}{2} ;-R(Z)^{2}\right)\right. \\
- & \left.R_{0}{ }_{2} F_{1}\left(\frac{1}{2}, \frac{-1+n}{2 n} ; \frac{3}{2} ;-R_{0}^{2}\right)\right\} .
\end{aligned}
$$

Substituting Eqs. (A.11) and (A.8) into $U(Z)=$ $Q(Z)+E(Z)$, we have:

$$
\begin{aligned}
& U(Z)=\eta(s) \frac{A^{X}}{\left(A^{X}\right)^{2}+\left(A^{Y}\right)^{2}} \frac{n}{n+1} \\
&\left\{\left[\left(A^{X} Z+B\right)^{2}+\left(A^{Y} Z+B^{\prime}\right)^{2}\right]^{(n+1) / 2 n}\right. \\
&- {\left.\left[(B)^{2}+\left(B^{\prime}\right)^{2}\right]^{(n+1) / 2 n}\right\} } \\
&- \frac{A^{Y}\left(\left|A^{X} B^{\prime}-A^{Y} B\right|\right)^{(n+1) / n}}{\left[\left(A^{X}\right)^{2}+\left(A^{Y}\right)^{2}\right]^{(3 n+1) / 2 n}} \\
&\left\{R(Z){ }_{2} F_{1}\left(\frac{1}{2}, \frac{-1+n}{2 n} ; \frac{3}{2} ;-R(Z)^{2}\right)\right. \\
&\left.-R_{0}{ }_{2} F_{1}\left(\frac{1}{2}, \frac{-1+n}{2 n} ; \frac{3}{2} ;-R_{0}^{2}\right)\right\} . \\
&(\mathrm{A} .12
\end{aligned}
$$

Similarly, for $V(Z)$, we have:

$$
\begin{aligned}
V(Z)= & \frac{A^{Y}}{\left(A^{X}\right)^{2}+\left(A^{Y}\right)^{2}} \frac{n}{n+1} \\
& \left\{\left[\left(A^{X} Z+B\right)^{2}+\left(A^{Y} Z+B^{\prime}\right)^{2}\right]^{(n+1) / 2 n}\right. \\
- & {\left.\left[(B)^{2}+\left(B^{\prime}\right)^{2}\right]^{(n+1) / 2 n}\right\} } \\
+ & \frac{A^{X}\left(\left|A^{X} B^{\prime}-A^{Y} B\right|\right)^{(n+1) / n}}{\left[\left(A^{X}\right)^{2}+\left(A^{Y}\right)^{2}\right]^{(3 n+1) / 2 n}} \\
& \left\{R(Z){ }_{2} F_{1}\left(\frac{1}{2}, \frac{-1+n}{2 n} ; \frac{3}{2} ;-R(Z)^{2}\right)\right. \\
& \left.-R_{0}{ }_{2} F_{1}\left(\frac{1}{2}, \frac{-1+n}{2 n} ; \frac{3}{2} ;-R_{0}^{2}\right)\right\} .
\end{aligned}
$$

\section{Biographies}

Ramin Nasehi received his BSc and MSc degrees in Mechanical Engineering from Isfahan University of Technology. His MSc thesis entitled "Analysis of a Thin Film of Non-Newtonian Fluid on a Surface" was under the supervision of Professor Ebrahim Shirani and developed an analytical relation for slow motion thin liquid films on a surface for non-Newtonian fluids.

Ebrahim Shirani is Emeritus Professor of Mechanical Engineering at Isfahan University of Technology. He is also Vice President for Research at FIT, Fellow of Academy of Science, Editor-in-Chief of JAFM, Associate Editor of African Review of Physics, and Senior Associate of ICTP. Professor Shirani also cooperates with Scientia Iranica Journal. 